\title{
Spectral triples and Gibbs measures for expanding maps on Cantor sets
}

\author{
Richard Sharp
}

\begin{abstract}
Let $T: \Lambda \rightarrow \Lambda$ be an expanding map on a Cantor set. For each suitably normalized Hölder continuous potential, we construct a spectral triple from which one may recover the associated Gibbs measure as a noncommutative measure.
\end{abstract}

Mathematics Subject Classification (2010). Primary 37D20 37D35 46L55 58B34; Secondary 37A55 46L51.

Keywords. Spectral triple, Dixmier trace, expanding map, Gibbs measure, Cantor set.

\section{Introduction}

In the 1990s, Connes introduced the concept of a spectral triple as a fundamental object in noncommutative geometry [7], [8], [9], [24], giving a "space-free" description of many geometric phenomena. The notion is very flexible and appropriate choices allow one to recover the volume measure and metric on a Riemannian spin manifold [7], [8] and also, for example, the Hausdorff measure on certain fractal sets [7], [12], [13], [14]. In the fractal case, the starting point is Connes's construction of a spectral triple for a Cantor subset of the real line, from which (using ideas of Lapidus and Pomerance [16]) the Minkowski content may be recovered. For simple self-similar sets, the Hausdorff measure may also be obtained and Guido and Isola generalize these ideas to certain fractal subsets of $\mathbb{R}^{n}$. (See [5], [6], [20] for other approaches and [18] for a more general construction valid for any compact metric space.) Furthermore, Falconer and Samuel [11] have modified this construction to describe multifractal phenomena.

The purpose of this paper is to show that, for a class of expanding maps, certain important measures, called Gibbs measures, which arise in the ergodic theory of hyperbolic dynamical systems, may be obtained as noncommutative measures from an appropriate spectral triple. Specifically, our dynamical systems will be expanding maps conjugate to a subshift of finite type (not necessarily a full shift), so that, in particular, the (maximal) invariant set is a Cantor set. After this paper was written, we learned that Samuel had obtained a very similar result in his thesis [22]. We will give a little more detail on his work following Theorem 2.1 below. 
We shall we shall now fix some notation. Let $\Lambda$ be a compact subset of a smooth Riemannian manifold $M$ and let $T: \Lambda \rightarrow \Lambda$ be a $C^{1}$ expanding map which is topologically conjugate to a mixing one-sided subshift of finite type $\sigma: \Sigma_{A}^{+} \rightarrow \Sigma_{A}^{+}$. (See Section 3 below for precise definitions.) In particular, $\Lambda$ is a Cantor set. The purpose of this condition is to ensure that so-called "locally constant" functions on $\Lambda$ are contained in $C(\Lambda, \mathbb{C})$.

Let $\mathcal{M}_{T}$ denote the set of $T$-invariant probability measures on $\Lambda$. This is a large set but we may single out the so-called Gibbs measures (or equilibrium measures) associated to Hölder continuous potentials as being of particular importance. These are defined as follows. Let $\psi: \Lambda \rightarrow \Lambda$ be a Hölder continuous function. Then the Gibbs measure for $\psi$ is the unique $\mu \in \mathcal{M}_{T}$ for which

$$
h_{T}(\mu)+\int \psi d \mu=\sup _{m \in \mathcal{M}_{T}}\left(h_{T}(m)+\int \psi d m\right) \text {, }
$$

where $h_{T}(m)$ denotes the entropy of $T$ with respect to $m$. A general feature of hyperbolic dynamical systems is that averages of weighted local data (e.g. sums of observables over sets of orbits) give global information (e.g. the average of an observable with respect to an invariant measure) [2], [19], [23] and Gibbs measures may be obtained in this way. (Very roughly, weighting by the exponentials of sums of an observable $\psi$ gives the Gibbs measure for $\psi$.) However, this local to global property also motivates the definition of a Dirac operator, adapted from those in [11], [12], [13], [14], and allows us to obtain a noncommutative integral from its spectrum. In Theorem 2.1 below, we show that this noncommutative integral agrees up to an explicit factor with the integral with respect the Gibbs measure. We begin by defining a spectral triple [7], [24].

Definition 1.1. A spectral triple is a triple $(H, A, D)$, where

(i) $H$ is a Hilbert space;

(ii) $A$ is a $C^{*}$-algebra equipped with a faithful representation $\pi: A \rightarrow B(H)$ (the bounded linear operators on $H$ );

(iii) $D$ is an essentially self-adjoint unbounded linear operator on $H$ with compact resolvent and such that $\{f \in A:\|[D, \pi(f)]\|<+\infty\}$ is dense in $A$, where $[D, \pi(f)]: H \rightarrow H$ is the commutator operator $[D, \pi(f)](\xi)=D \pi(f)(\xi)-$ $\pi(f) D(\xi)$. This $D$ is called a Dirac operator.

We shall define spectral triples associated to Hölder continuous potentials on $\Lambda$, adapting the constructions of Connes [7], Guido and Isola [12], [13], [14]) and Falconer and Samuel [11]. As above, let $\sigma: \Sigma_{A}^{+} \rightarrow \Sigma_{A}^{+}$be the subshift of finite type topologically conjugate to the expanding map $T: \Lambda \rightarrow \Lambda$, where the symbol set is $\{1, \ldots, k\}$ and $A$ is a zero-one transition matrix. (See Section 3 for a complete definition.) We shall write $p: \Sigma_{A}^{+} \rightarrow \Lambda$ for the conjugating homeomorphism. In the 
interests of readability, we will systematically abuse notation by writing $f(x)$ instead of $f(p(x))$, whenever $f \in C(\Lambda, \mathbb{C})$ and $x \in \Sigma_{A}^{+}$.

An ordered $n$-tuple $\left(w_{1}, \ldots, w_{n}\right)$, with $w_{m} \in\{1, \ldots, k\}, m=1, \ldots, n$, is called an allowed word of length $n$ if $A\left(w_{m}, w_{m+1}\right)=1$ for $m=1, \ldots, n-1$. Let $W_{n}$ denote the set of allowed words of length $n$ and let

$$
W^{*}=\bigcup_{n=1}^{\infty} W_{n}
$$

For $w=\left(w_{1}, \ldots, w_{n}\right) \in W_{n}$, we write

$$
[w]=\left\{x=\left(x_{n}\right)_{n=1}^{\infty} \in \Sigma_{A}^{+}: x_{m}=w_{m}, m=1, \ldots, n\right\}
$$

and $\mathrm{t}(w)=w_{n}$. For $w \in W_{n}$ and $x \in \Sigma_{A}^{+}$then $w x$ will denote the sequence defined by

$$
(w x)_{m}= \begin{cases}w_{m} & \text { if } 1 \leq m \leq n, \\ x_{m-n} & \text { if } m \geq n+1 .\end{cases}
$$

Clearly, $w x \in \Sigma_{A}^{+}$if and only if $A\left(\mathrm{t}(w), x_{1}\right)=1$. For each $j \in\{1, \ldots, k\}$, choose a sequence $x^{j} \in \Sigma_{A}^{+}$and distinct sequences $y^{j}, z^{j} \in \Sigma_{A}^{+}$such that $j x^{j}, j y^{j}, j z^{j} \in$ $\Sigma_{A}^{+}$.

Now we can define a spectral triple associated to a continuous potential $\phi: \Lambda \rightarrow$ $\mathbb{R}$. Our Hilbert space will be

$$
H=\ell^{2}\left(W^{*}\right) \oplus \ell^{2}\left(W^{*}\right) \subset \bigoplus_{w \in W^{*}} \mathbb{C} \oplus \mathbb{C},
$$

where we write a typical element as

$$
\xi=\bigoplus_{w \in W^{*}}\left(\begin{array}{l}
\xi_{1}(w) \\
\xi_{2}(w)
\end{array}\right)
$$

and our $C^{*}$-algebra will be $A=C(\Lambda, \mathbb{C})$. We define a *-representation $\pi: A \rightarrow$ $B(H)$ by setting $\pi(f)$ to be the multiplication operator

$$
\pi(f)\left(\bigoplus_{w \in W^{*}}\left(\begin{array}{l}
\xi_{1}(w) \\
\xi_{2}(w)
\end{array}\right)\right)=\bigoplus_{w \in W^{*}}\left(\begin{array}{l}
f\left(w y^{\mathrm{t}(w)}\right) \xi_{1}(w) \\
f\left(w z^{\mathrm{t}(w)}\right) \xi_{2}(w)
\end{array}\right),
$$

We define $D_{\phi}: H \rightarrow H$ by

$$
\begin{aligned}
D_{\phi}\left(\bigoplus_{w \in W^{*}}\left(\begin{array}{l}
\xi_{1}(w) \\
\xi_{2}(w)
\end{array}\right)\right) & =\bigoplus_{n=1}^{\infty} \bigoplus_{w \in W_{n}} e^{\phi^{n}\left(w x^{\mathrm{t}(w)}\right)}\left(\begin{array}{ll}
0 & 1 \\
1 & 0
\end{array}\right)\left(\begin{array}{l}
\xi_{1}(w) \\
\xi_{2}(w)
\end{array}\right) \\
& =\bigoplus_{n=1}^{\infty} \bigoplus_{w \in W_{n}} e^{\phi^{n}\left(w x^{\mathrm{t}(w)}\right)}\left(\begin{array}{l}
\xi_{2}(w) \\
\xi_{1}(w)
\end{array}\right),
\end{aligned}
$$

where $\phi^{n}:=\phi+\phi \circ T+\cdots+\phi \circ T^{n-1}$. We have the following theorem. 
Theorem 1.2. For any continuous function $\phi: \Lambda \rightarrow \mathbb{R},\left(H, A, D_{\phi}\right)$ is a spectral triple.

The main result of the paper is that, when $\phi$ is Hölder continuous and is suitably normalized, we may recover the Gibbs measure for $-\phi$ from the operators $\pi(f)\left|D_{\phi}\right|^{-1}$ via a singular trace. (The choice of sign is for notational convenience.) In the next section, we introduce the ideas needed to explain this statement and then state our main theorem. In Section 3, we discuss some material on expanding maps, subshifts of finite type and transfer operators. In Section 4, we prove Theorem 1.2. In Section 5, we complete the paper by proving our result on noncommutative measures and Gibbs measures, Theorem 2.1.

\section{Singular traces and noncommutative measures}

In order to state our main result, we need to briefly discuss the theory of singular traces of compact operators. For more details, see [1] or [13]. Let $B(H)$ denote the algebra of bounded linear operators on a Hilbert space $H$ and let $K(H)$ denote the ideal of compact operators. A singular trace on a two-sided ideal $I \subset K(H)$ is a positive linear functional $\tau: I \rightarrow \mathbb{R}$ such that $\tau$ is unitary invariant (the trace property) and vanishes on finite rank operators.

The most important singular traces are the so-called Dixmier traces [10]. These are defined on an ideal $I=\mathscr{L}^{1, \infty}(H)$, the Dixmier ideal, given by

$$
\mathscr{L}^{1, \infty}(H)=\left\{A \in K(H): \limsup _{n \rightarrow+\infty} \frac{1}{\log n} \sum_{k=1}^{n} a_{k}<+\infty\right\},
$$

where $\left\{a_{n}\right\}_{n=1}^{\infty}$ denote the eigenvalues of $|A|:=\sqrt{A^{*} A}$, written in decreasing order. Then a Dixmier trace is a singular trace $\tau_{\omega}$ on $\mathscr{L}^{1, \infty}(H)$ defined, for a positive operator $A$, by

$$
\tau_{\omega}(A)=\omega-\lim \frac{1}{\log n} \sum_{k=1}^{n} a_{k},
$$

where this is a generalized limit corresponding to a state $\omega$ on $l^{\infty}$, and extended to $\mathscr{L}^{1, \infty}(H)$ by linearity. If the limit

$$
\lim _{n \rightarrow+\infty} \frac{1}{\log n} \sum_{k=1}^{n} a_{k}
$$

exists then we say that $A$ is measurable and call the value of the limit the noncommutative integral of $A$. (There are more general definitions of the Dixmier trace - see, for example, Chapter IV, $§ 2 . \beta$ of [7], [17] or Chapter 5 of [24]. Correspondingly, 
there are more general definitions of measurability. It is shown in [17] that these are equivalent to the definition given here.)

Consider the spectral triple $\left(H, A, D_{\phi}\right)$ defined in the previous section. We will now suppose that $\phi: \Lambda \rightarrow \mathbb{R}$ is Hölder continuous. We say that $-\phi$ is normalized if

$$
\sum_{T y=x} e^{-\phi(y)}=1
$$

for all $x \in \Lambda$. (As we shall see in Section 3, any real-valued Hölder continuous function may be normalized by adding a constant and a function of the form $u \circ T-u$, with $u \in C(\Lambda, \mathbb{R})$, and this operation does not change the Gibbs measure.) Then, for $f \in C(\Lambda, \mathbb{C})$, the operator $\pi(f)\left|D_{\phi}\right|^{-1}$ is given by the formula

$$
\pi(f)\left|D_{\phi}\right|^{-1}\left(\bigoplus_{w \in W^{*}}\left(\begin{array}{l}
\xi_{1}(w) \\
\xi_{2}(w)
\end{array}\right)\right)=\bigoplus_{n=1}^{\infty} \bigoplus_{w \in W_{n}} e^{-\phi^{n}\left(w x^{\mathrm{t}(w)}\right)}\left(\begin{array}{l}
f\left(w y^{\mathrm{t}(w)}\right) \xi_{1}(w) \\
f\left(w z^{\mathrm{t}(w)}\right) \xi_{2}(w)
\end{array}\right) .
$$

Theorem 2.1. Suppose that $\phi: \Lambda \rightarrow \mathbb{R}$ is a Hölder continuous function and that $-\phi$ is normalized. Then, for any $f \in C(\Lambda, \mathbb{C})$,

(i) $\pi(f)\left|D_{\phi}\right|^{-1} \in \mathscr{L}^{1, \infty}(H)$;

(ii) $\pi(f)\left|D_{\phi}\right|^{-1}$ is measurable and

$$
\tau_{\omega}\left(\pi(f)\left|D_{\phi}\right|^{-1}\right)=c_{\phi} \int f d \mu
$$

where $\mu$ is the Gibbs measure for $-\phi$ and where

$$
c_{\phi}=\frac{2}{\int \phi d \mu} \sum_{j=1}^{k} \sum_{T x=x^{j}} e^{-\phi(x)} \chi_{j}(x)
$$

with $\chi_{j}$ the indicator function of the set $p([j])$.

Remark 2.2. As we noted in the introduction, a result similar to Theorem 2.1 has been obtained by Samuel [22]. A significant difference is that he requires the potential $\phi$ to be non-arithmetic, i.e., that the sums of $\phi$ around periodic orbits do not all lie in a single discrete subgroup of $\mathbb{R}$. This restriction is needed for the renewal theory approach he uses. Thus, for example, his results do not cover the measure of maximal entropy. A particularly attractive feature of his work is that he is able to explicitly calculate the Dixmier trace associated to the constant function 1, i.e. $\tau_{\omega}\left(\left|D_{\phi}\right|^{-1}\right)$, is equal to the reciprocal of the entropy of $\mu$ and he identifies this as a noncommutative volume. 


\section{Expanding maps and subshifts of finite type}

We begin the section by defining subshifts of finite type. Let $A$ be a $k \times k$ matrix whose entries are all either zero or one. We define the (one-sided) shift space

$$
\Sigma_{A}^{+}=\left\{\left(x_{n}\right)_{n=1}^{\infty} \in \prod_{n=1}^{\infty}\{1, \ldots, k\}: A\left(x_{n}, x_{n+1}\right)=1 \text { for all } n \geq 1\right\}
$$

and the (one-sided) subshift of finite type $\sigma: \Sigma_{A}^{+} \rightarrow \Sigma_{A}^{+}$by $(\sigma x)_{n}=x_{n+1}$. We give $\{1, \ldots, k\}$ the discrete topology, $\prod_{n=1}^{\infty}\{1, \ldots, k\}$ the product topology and $\Sigma_{A}^{+}$the subspace topology. A compatible metric is given by

$$
d\left(\left(x_{n}\right)_{n=1}^{\infty},\left(y_{n}\right)_{n=1}^{\infty}\right)=\sum_{n=1}^{\infty} \frac{1-\delta_{x_{n} y_{n}}}{2^{n}},
$$

where $\delta_{i j}$ is the Kronecker symbol.

We say that the matrix $A$ is irreducible if, for each $(i, j)$, there exists $n(i, j) \geq 1$ such that $A^{n(i, j)}(i, j)>0$ and aperiodic if there exists $n \geq 1$ such that, for each $(i, j), A^{n}(i, j)>0$. The latter statement is equivalent to $\sigma: \Sigma_{A}^{+} \rightarrow \Sigma_{A}^{+}$being topologically mixing (i.e. that there exists $n \geq 1$ such that for any two non-empty open sets $U, V \subset \Sigma_{A}^{+}, \sigma^{-m}(U) \cap V \neq \varnothing$, for all $\left.m \geq n\right)$.

Let $M$ be a compact connected smooth Riemannian manifold and suppose that $\Lambda \subset U \subset M$ with $\Lambda$ compact and $U$ open. Let $T: U \rightarrow M$ be a $C^{1}$ map. Suppose that

(i) there exists $\lambda>1$ such that $\left\|D T_{x}\right\| \geq \lambda$ for all $x \in U$;

(ii) $\Lambda=\bigcap_{n=0}^{\infty} T^{-n} U$;

(iii) $T$ is topologically mixing.

If $T$ satisfies (i), (ii) and (iii) then we refer to $T: \Lambda \rightarrow \Lambda$ as an expanding map and we can find a mixing one-sided subshift of finite type $\sigma: \Sigma_{A}^{+} \rightarrow \Sigma_{A}^{+}$and a Hölder continuous map $p: \Sigma_{A}^{+} \rightarrow \Lambda$ which semi-conjugates $T$ and $\sigma$. Furthermore, the map is "nearly" a homeomorphism. Here, however, we impose the additional condition that $p$ is, in fact, a homeomorphism and assume that

(iv) $T: \Lambda \rightarrow \Lambda$ is topologically conjugate to a mixing one-sided subshift of finite type $\sigma: \Sigma_{A}^{+} \rightarrow \Sigma_{A}^{+}$.

In particular, (iv) implies that $\Lambda$ is a Cantor set.

Assumption (iv) gives $\Lambda$ a natural grading. In particular, for each $n \geq 1$, we may write $\Lambda$ as a disjoint union

$$
\Lambda=\bigcup_{w \in W_{n}} p([w]) .
$$

We will say that a function $f: \Lambda \rightarrow \mathbb{C}$ is locally constant if, for some $n \geq 1, f$ is constant on each set $p([w]), w \in W_{n}$. We shall write $\operatorname{LC}(\Lambda)$ for the set of all locally constant functions on $\Lambda$. Clearly, $\operatorname{LC}(\Lambda)$ a uniformly dense subalgebra of $C(\Lambda, \mathbb{C})$. 
We shall also consider some larger subalgebras of $C(\Lambda, \mathbb{C})$. For $\alpha>0$, we shall let $C^{\alpha}(\Lambda, \mathbb{C})$ denote the space of $\alpha$-Hölder continuous functions on $\Lambda$, i.e., the set of functions $g: \Lambda \rightarrow \mathbb{C}$ satisfying

$$
|g|_{\alpha}:=\sup _{x \neq y} \frac{|g(x)-g(y)|}{d(x, y)^{\alpha}}<+\infty .
$$

This is a Banach space with respect to the norm $\|\cdot\|_{\alpha}=\|\cdot\|_{\infty}+|\cdot|_{\alpha}$. Clearly, for any $\alpha>0$,

$$
\operatorname{LC}(\Lambda) \subset C^{\alpha}(\Lambda, \mathbb{C}) \subset C(\Lambda, \mathbb{C}) .
$$

\section{Gibbs states and transfer operators}

In this section we shall discuss some of the ergodic theory associate to the map $T: \Lambda \rightarrow \Lambda$. The main references are [4] and [19], where this theory is developed for subshifts of finite type. The symbolic dynamics described in the preceding section allows the results to be immediately transferred to expanding maps. As above, we shall write $\mathcal{M}_{T}$ for the space of $T$-invariant probability measures. Given $m \in \mathcal{M}_{T}$, we write $h_{T}(m) \geq 0$ for the entropy of $T$ as a measure preserving transformation of $(\Lambda, m)$ (see [25] for the definition). For a continuous function $\psi: \Lambda \rightarrow \mathbb{R}$, we define its pressure $P(\psi)$ by

$$
P(\psi)=\sup _{m \in \mathcal{M}_{T}}\left(h_{T}(m)+\int \psi d m\right) .
$$

If $\psi$ is Hölder continuous, then there is a unique probability measure $\mu$, called the Gibbs measure (or equilibrium measure) for $\psi$, for which this supremum is realized [2], [4], [19]. by

Given $\psi \in C(\Lambda, \mathbb{R})$, we define the transfer operator $L_{\psi}: C(\Lambda, \mathbb{C}) \rightarrow C(\Lambda, \mathbb{C})$

$$
L_{\psi} g(x)=\sum_{T y=x} e^{\psi(y)} g(y) .
$$

A key element of our approach will be to relate the eigenvalue asymptotics of our Dirac operators to the spectral properties of transfer operators. For this approach to work, we shall need to find a space of which $L_{\psi}$ acts quasi-compactly.

If $\psi \in C^{\alpha}(\Lambda, \mathbb{R})$ then $L_{\psi}: C^{\alpha}(\Lambda, \mathbb{C}) \rightarrow C^{\alpha}(\Lambda, \mathbb{C})$. The basic spectral properties of $L_{\psi}$ on this space are contained in the following result, which is Ruelle's generalization of the classical Perron-Frobenius Theorem for non-negative matrices).

Proposition 4.1 ([2], [4], [19], [21]). If $\psi \in C^{\alpha}(\Lambda, \mathbb{R})$ then $L_{\psi}: C^{\alpha}(\Lambda, \mathbb{C}) \rightarrow$ $C^{\alpha}(\Lambda, \mathbb{C})$ has a simple eigenvalue equal to $e^{P(\psi)}$ with the rest of the spectrum contained in a disk $\left\{z \in \mathbb{C}:|z| \leq \theta e^{P(\psi)}\right\}$, for some $0<\theta<1$. Furthermore, there exist 
(i) a strictly positive eigenfunction $h \in C^{\alpha}(\Lambda, \mathbb{R})$ such that $L_{\psi} h=e^{P(\psi)} h$; and

(ii) an eigenmeasure $v \in C(\Lambda, \mathbb{R})^{*}$ such that $L_{\psi}^{*} v=e^{P(\psi)} \nu$.

If $v$ is chosen to be a probability measure and the eigenfunction $h$ is chosen so that $\int h d v=1$ then $\mu=h v$ is the Gibbs measure for $\psi$.

Corollary 4.2. There exists $\lambda_{\psi}<e^{P(\psi)}$ such that, for any $f \in C^{\alpha}(\Lambda, \mathbb{C})$, we have

$$
L_{\psi}^{n} f=\left(\int f d \nu\right) h e^{n P(\psi)}+O\left(\lambda_{\psi}^{n}\right) .
$$

Proof. We recall the following basic fact from spectral theory (see, for example, [3] or [15]). Let $L: B \rightarrow B$ be a bounded linear operator on a Banach space $B$ with spectrum $\operatorname{spec}(L)=\Sigma \subset \mathbb{C}$. If $\Sigma$ can be decomposed into two disjoint non-empty sets $\Sigma_{1}$ and $\Sigma_{2}$ and if $\gamma$ is a simple closed curve which is disjoint from $\Sigma$ and which has $\Sigma_{1}$ in its interior and $\Sigma_{2}$ in its exterior then $\Pi: B \rightarrow B$ defined by

$$
\Pi=\frac{1}{2 \pi i} \int_{\gamma}(z-L)^{-1} d z,
$$

is a projection (i.e. $\|\Pi\|=1$ and $\Pi^{2}=\Pi$ ). Moreover, $B=B_{1} \oplus B_{2}$, where $B_{1}=\Pi_{1}(B)$ and $B_{2}=(I-\Pi)(B)$ are closed and $L$-invariant subspaces with $\operatorname{spec}\left(L \mid B_{1}\right)=\Sigma_{1}$ and $\operatorname{spec}\left(L \mid B_{2}\right)=\Sigma_{2}$.

Now consider the operator $L_{\psi}: C^{\alpha}(\Lambda, \mathbb{C}) \rightarrow C^{\alpha}(\Lambda, \mathbb{C})$. By Theorem 4.1, we may decompose its spectrum into $\Sigma_{1}=\left\{e^{P(\psi)}\right\}$ and a disjoint set $\Sigma_{2}$. Thus, we may decompose the operator $L_{\psi}$ as a sum

$$
L_{\psi}^{n}=L_{\psi}^{n} \Pi+L_{\psi}^{n}(I-\Pi)=e^{n P(\psi)} v(\cdot) h+L_{\psi}^{n}(I-\Pi),
$$

where $\Pi_{1}=v(\cdot) h$ is the projection onto the eigenspace spanned by $e^{P(\psi)}$. Furthermore, since $e^{P(\psi)}$ is strictly maximal in modulus, we have

$$
\lim _{n \rightarrow+\infty}\left\|L_{\psi}^{n}(I-\Pi)\right\|^{1 / n}=\sup \left\{|z|: z \in \Sigma_{2}\right\}<e^{P(\psi)} .
$$

Choosing $\lambda_{\psi}$ slightly larger than $\lim _{n \rightarrow+\infty}\left\|L_{\psi}^{n}(I-\Pi)\right\|^{1 / n}$ completes the proof.

Corollary 4.3. The quantities $e^{P(\psi)}, h$ and $v$ in Theorem 4.1 all depend analytically on $\psi$.

Proof. First we note that $L_{\psi}$ depends analytically on $\psi$. The result is then a standard consequence of the fact that $e^{P(\psi)}$ is an isolated simple eigenvalue for $L_{\psi}$ [3], [15]. 
Recall that we defined a function $-\phi$ to be normalized if, for all $x \in \Lambda$,

$$
\sum_{T y=x} e^{-\phi(y)}=1
$$

In particular, this condition implies that $-\phi$ is strictly negative. We may rewrite this condition in terms of transfer operators as $L_{-\phi} 1=1$. The following consequence of Theorem 4.1 shows that, given a Hölder continuous function, we may find another which is normalized and which had the same Gibbs measure.

Corollary 4.4. Suppose that $\psi, h, v, \mu$ are as in Theorem 4.1. Then

$$
-\phi:=\psi+\log h-\log h \circ T-P(\psi) \in C^{\alpha}(\Lambda, \mathbb{R})
$$

is normalized, $L_{-\phi}^{*} \mu=\mu$ and $\mu$ is the Gibbs state $-\phi$.

Proof. Since $h>0,-\phi$ is well defined. For any $m \in \mathcal{M}_{T}$,

$$
h_{T}(m)+\int-\phi d m=h_{T}(m)+\int \psi d m-P(\psi),
$$

so it follows that $P(-\phi)=0$ and that $\mu$ is the Gibbs measure for $-\phi$. We also have

$$
\begin{aligned}
L_{-\phi} 1(x) & =\sum_{T y=x} e^{-\phi(y)}=\sum_{T y=x} e^{\psi(y)+\log h(y)-\log h(T y)-P(\psi)} \\
& =\frac{e^{-P(\psi)}}{h(x)} \sum_{T y=x} e^{\psi(y)} h(y)=\frac{e^{-P(\psi)}}{h(x)} L_{\psi} h(x) \\
& =\frac{e^{-P(\psi)}}{h(x)} e^{P(\psi)} h(x)=1,
\end{aligned}
$$

so $-\phi$ is normalized. By Theorem $4.1, L_{-\phi}^{*} \mu=\mu$.

To prove Theorem 2.1, we shall need to consider a family of transfer operators $L_{-t \phi}$, for $t \in \mathbb{R}$. By Theorem 4.1, these will have a maximal eigenvalue equal to $e^{P(-t \phi)}$. We end the section with a result on the regularity and derivative of the function $t \mapsto P(-t \phi)$.

Lemma 4.5. The function $t \mapsto P(-t \phi)$ is real-analytic and strictly decreasing. Furthermore,

$$
\left.\frac{d P(-t \phi)}{d t}\right|_{t=1}=-\int \phi d \mu,
$$

where $\mu$ is the Gibbs measure for $-\phi$. 


\section{Proof of Theorem 1.2}

In this section we proof that the $\left(H, A, D_{\phi}\right)$ we have constructed is a spectral triple. The key point is that the locally constant functions give a dense subalgebra of $C(\Lambda, \mathbb{C})$ on which $\left\|\left[D_{\phi}, \pi(f)\right]\right\|$ is finite.

Proof of Theorem 1.2. Suppose that $f_{1}, f_{2} \in C(\Lambda, \mathbb{C})$ and that $\pi\left(f_{1}\right)=\pi\left(f_{2}\right)$. Then, in particular, by definition, for each $w \in W^{*}, f_{1}\left(w y^{\mathrm{t}(w)}\right)=f_{2}\left(w y^{\mathrm{t}(w)}\right)$. The set $\left\{w y^{\mathrm{t}(w)}: w \in W^{*}\right\}$ is dense in $\Sigma_{A}^{+}$and thus the set $p\left(\left\{w y^{\mathrm{t}(w)}: w \in W^{*}\right\}\right)$ is dense in $\Lambda$. Hence $f_{1}=f_{2}$ and $\pi: C(\Lambda, \mathbb{C}) \rightarrow B(H)$ is faithful.

It is clear from its definition that $D_{\phi}$ is self-adjoint. The eigenvalues of $D_{\phi}$ are the numbers

$$
\bigcup_{n=1}^{\infty}\left\{e^{\phi^{n}\left(w x^{\mathrm{t}(w)}\right)}: w \in W_{n}\right\}
$$

(counted with the appropriate multiplicity). In particular, 0 is not an eigenvalue. Thus, the resolvent of $D_{\phi}$ is compact provided $D_{\phi}^{-1}$ is compact and it is clear that $D_{\phi}^{-1}$, defined by

$$
D_{\phi}^{-1}\left(\bigoplus_{w \in W^{*}}\left(\begin{array}{l}
\xi_{1}(w) \\
\xi_{2}(w)
\end{array}\right)\right)=\bigoplus_{n=1}^{\infty} \bigoplus_{w \in W^{*}} e^{-\phi^{n}\left(w x^{\mathrm{t}(w)}\right)}\left(\begin{array}{ll}
0 & 1 \\
1 & 0
\end{array}\right)\left(\begin{array}{l}
\xi_{1}(w) \\
\xi_{2}(w)
\end{array}\right)
$$

is a compact operator. For $f \in C(\Lambda, \mathbb{C})$,

$$
\begin{aligned}
& {\left[D_{\phi}, \pi(f)\right]\left(\bigoplus_{w \in W^{*}}\left(\begin{array}{l}
\xi_{1}(w) \\
\xi_{2}(w)
\end{array}\right)\right)} \\
& =\bigoplus_{w \in W^{*}}\left(f\left(w y^{\mathrm{t}(w)}\right)-f\left(w z^{\mathrm{t}(w)}\right)\right) e^{\phi^{n}\left(w x^{\mathrm{t}(w)}\right)}\left(\begin{array}{cc}
0 & -1 \\
1 & 0
\end{array}\right)\left(\begin{array}{l}
\xi_{1}(w) \\
\xi_{2}(w)
\end{array}\right) \\
& =\bigoplus_{w \in W^{*}}\left(f\left(w y^{\mathrm{t}(w)}\right)-f\left(w z^{\mathrm{t}(w)}\right)\right) e^{\phi^{n}\left(w x^{\mathrm{t}(w)}\right)}\left(\begin{array}{c}
-\xi_{2}(w) \\
\xi_{1}(w)
\end{array}\right) .
\end{aligned}
$$

Let $A_{0}=\mathrm{LC}(\Lambda)$, the subalgebra of locally constant functions on $\Lambda$. Recall that $A_{0}$ is dense in $A$. If $f \in A_{0}$ then there exists $N \geq 1$ such that

$$
f\left(w y^{\mathrm{t}(w)}\right)=f\left(w z^{\mathrm{t}(w)}\right) \quad \text { for all } w \in \bigcup_{n=N+1}^{\infty} W_{n} .
$$

Then

$$
\begin{aligned}
\left\|[D, \pi(f)]\left(\bigoplus_{w \in W^{*}}\left(\begin{array}{l}
\xi_{1}(w) \\
\xi_{2}(w)
\end{array}\right)\right)\right\|_{2}^{2} \\
\quad=\sum_{n=1}^{\infty} \sum_{w \in W_{n}}\left(f\left(w y^{\mathrm{t}(w)}\right)-f\left(w z^{\mathrm{t}(w)}\right)\right)^{2} e^{2 \phi^{n}\left(w x^{\mathrm{t}(w)}\right)}\left(\left(-\xi_{2}(w)\right)^{2}+\xi_{1}(w)^{2}\right)
\end{aligned}
$$




$$
\begin{aligned}
& \leq 2\|f\|_{\infty} e^{2 N\|\phi\|_{\infty}} \sum_{n=1}^{N} \sum_{w \in W_{n}}\left(\left(-\xi_{2}(w)\right)^{2}+\xi_{1}(w)^{2}\right) \\
& \leq 2\|f\|_{\infty} e^{2 N\|\phi\|_{\infty}}\|\xi\|_{2}^{2} .
\end{aligned}
$$

Hence $\|[D, \pi(f)]\|<+\infty$.

\section{Proof of Theorem 2.1}

We will use the following version of the Hardy-Littlewood Tauberian Theorem. (See [7], Chapter IV, §2. $\beta$, Proposition 4.)

Lemma 6.1. Suppose that $A \in K(H)$ is a positive operator with eigenvalues $\left\{a_{n}\right\}_{n=1}^{\infty}$ (arranged in decreasing order) and that $A \in \mathscr{L}^{1, \infty}(H)$. Write

$$
\zeta(t)=\sum_{n=1}^{\infty} a_{n}^{t}
$$

Then

$$
\lim _{t \rightarrow 1+}(t-1) \zeta(t)=L
$$

if and only if

$$
\lim _{n \rightarrow+\infty} \frac{1}{\log n} \sum_{k=1}^{n} a_{k}=L
$$

We will suppose for the moment that $f \in C^{\alpha}(\Lambda, \mathbb{R})$ and that $f \geq 0$, so that $\pi(f)\left|D_{\phi}\right|^{-1}$ is a positive operator. The eigenvalues of $\pi(f)\left|D_{\phi}\right|^{-1}$ are the numbers

$$
\bigcup_{n=1}^{\infty}\left\{f\left(w y^{\mathrm{t}(w)}\right) e^{-\phi^{n}\left(w x^{\mathrm{t}(w)}\right)}, f\left(w z^{\mathrm{t}(w)}\right) e^{-\phi^{n}\left(w x^{\mathrm{t}(w)}\right)}: w \in W_{n}\right\}
$$

(counted with multiplicity). We define a spectral zeta function

$$
\zeta_{f}(t)=\sum_{n=1}^{\infty} \sum_{w \in W_{n}}\left(\left(f\left(w y^{\mathrm{t}(w)}\right) e^{-\phi^{n}\left(w x^{\mathrm{t}(w)}\right)}\right)^{t}+\left(f\left(w z^{\mathrm{t}(w)}\right) e^{-\phi^{n}\left(w x^{\mathrm{t}(w)}\right)}\right)^{t}\right)
$$

and we also write

$$
\begin{aligned}
& \zeta_{f, x}(t)=\sum_{n=1}^{\infty} \sum_{w \in W_{n}}\left(f\left(w x^{\mathrm{t}(w)}\right) e^{-\phi^{n}\left(w x^{\mathrm{t}(w)}\right)}\right)^{t} \\
& \zeta_{f, y}(t)=\sum_{n=1}^{\infty} \sum_{w \in W_{n}}\left(f\left(w y^{\mathrm{t}(w)}\right) e^{-\phi^{n}\left(w x^{\mathrm{t}(w)}\right)}\right)^{t}
\end{aligned}
$$


and

$$
\zeta_{f, z}(t)=\sum_{n=1}^{\infty} \sum_{w \in W_{n}}\left(f\left(w z^{\mathrm{t}(w)}\right) e^{-\phi^{n}\left(w x^{\mathrm{t}(w)}\right)}\right)^{t} .
$$

In order to study these functions, it will be convenient to introduce another one which is easier to express in terms of transfer operators. Hence we define

$$
\eta_{f}(s)=\sum_{n=1}^{\infty} \sum_{w \in W_{n}} f\left(w x^{\mathrm{t}(w)}\right) e^{-s \phi^{n}\left(w x^{\mathrm{t}(w)}\right)} .
$$

Lemma 6.2. For $f \in C^{\alpha}(\Lambda, \mathbb{R})$ with $f>0, \eta_{f}(t)$ converges for $t>1$ and

$$
\lim _{t \rightarrow 1+}(t-1) \eta_{f}(t)=\left(\frac{\int f d \mu}{\int \phi d \mu}\right)\left(\sum_{j=1}^{k}\left(L_{-\phi} \chi_{j}\right)\left(x^{(j)}\right)\right) .
$$

Proof. Provided $\eta_{f}(t)$ converges, we may use the definitions of $x^{(j)}$ and $L_{-t \phi}$ to write

$$
\begin{aligned}
\eta_{f}(t) & =\sum_{n=1}^{\infty} \sum_{w \in W_{n}} f\left(w x^{\mathrm{t}(w)}\right) e^{-t \phi^{n}\left(w x^{\mathrm{t}(w)}\right)} \\
& =\sum_{n=1}^{\infty} \sum_{j=1}^{k} L_{-t \phi}^{n}\left(\chi_{j} \circ T^{n-1} f\right)\left(x^{j}\right) \\
& =\sum_{n=1}^{\infty} \sum_{j=1}^{k} L_{-t \phi}\left(\chi_{j} L_{-t \phi}^{n-1} f\right)\left(x^{j}\right) .
\end{aligned}
$$

By Theorem 4.1 and Lemma 4.5, for $t>1, L_{-t \phi}$ has spectral radius $e^{P(-t \phi)}<1$. Thus, using the spectral radius formula, it is easy to see that $\eta_{f}(t)$ converges. Furthermore, by Corollary 4.2, we have

$$
\begin{aligned}
\eta_{f}(t) & =\sum_{n=0}^{\infty} \sum_{j=1}^{k}\left(\int f d v_{t}\right) e^{n P(-t \phi)}\left(L_{-t \phi}\left(\chi_{j} h_{t}\right)\right)\left(x^{j}\right)+\sum_{n=0}^{\infty} q_{n}(t) \\
& =\left(\int f d v_{t}\right) \sum_{n=0}^{\infty} e^{n P(-t \phi)}\left(\sum_{j=1}^{k}\left(L_{-t \phi}\left(\chi_{j} h_{t}\right)\right)\left(x^{j}\right)\right)+\sum_{n=1}^{\infty} q_{n}(t) \\
& =\left(\int f d v_{t}\right) \frac{\sum_{j=1}^{k}\left(L_{-t \phi}\left(\chi_{j} h_{t}\right)\right)\left(x^{j}\right)}{1-e^{P(-t \phi)}}+\sum_{n=1}^{\infty} q_{n}(t),
\end{aligned}
$$

where $h_{t}$ and $v_{t}$ are the eigenfunction and eigenmeasure for $L_{-t \phi}$ given by Theorem 4.1 and where $q_{n}(t)=O\left(\lambda_{-t \phi}^{n}\right)$ (with $\left.\lambda_{-t \phi}<e^{P(-t \phi)}\right)$. Since 
(i) $t \mapsto e^{P(-t \phi)}, t \mapsto h_{t}$ and $t \mapsto v_{t}$ are all analytic;

(ii) $e^{P(-\phi)}=1, h_{1}=1$ and $v_{1}=\mu$; and

(iii)

$$
\left.\frac{d e^{P(-t \phi)}}{d t}\right|_{t=1}=-\int \phi d \mu
$$

we see that

$$
\eta_{f}(t)=\left(\frac{\int f d \mu}{\int \phi d \mu}\right)\left(\sum_{j=1}^{k}\left(L_{-\phi} \chi_{j}\right)\left(x^{j}\right)\right) \frac{1}{t-1}+a(t),
$$

where $a(t)$ is finite for $t \geq 1$.

Remark 6.3. In fact, the analyticity result in Corollary 4.3 enable one to deduce that, considered as a function of a complex variable $s, \eta_{f}(s)$ has a meromorphic extension to a neighbourhood of $s=1$, with a simple pole at $s=1$. Using the type of methods described in [19], [23], one can further show that $\eta_{f}(s)$ is analytic for $\operatorname{Re}(s)>1$ and that, provided the sums of $\phi$ around periodic orbits do not all lie in a discrete subgroup of $\mathbb{R}$, apart from the pole at $s=1, \eta_{f}(s)$ has an analytic extension to a neighbourhood of $\operatorname{Re}(s) \geq 1$.

Lemma 6.4. For $f \in C^{\alpha}(\Lambda, \mathbb{R})$ with $f>0, \zeta_{f, y}(t)$ and $\zeta_{f, z}(t)$ converge for $t>1$. Furthermore, we have

$$
\lim _{t \rightarrow 1+}(t-1) \zeta_{f, y}(t)=\lim _{t \rightarrow 1+}(t-1) \zeta_{f, z}(t)=\lim _{t \rightarrow 1+}(t-1) \eta_{f}(t) .
$$

Proof. First we shall show that it suffices to consider $\zeta_{f, x}(t)$. Note that, for $t>1$,

$$
\begin{aligned}
\left|\zeta_{f, x}(t)-\zeta_{f, y}(t)\right| & \leq \sum_{n=1}^{\infty} \sum_{w \in W_{n}}\left|f\left(w x^{\mathrm{t}(w)}\right)^{t}-f\left(w y^{\mathrm{t}(w)}\right)^{t}\right| e^{-t \phi^{n}\left(w x^{\mathrm{t}(w)}\right)} \\
& \leq t\|f\|_{\infty}^{t-1} \sum_{n=1}^{\infty} \sum_{w \in W_{n}}\left|f\left(w x^{\mathrm{t}(w)}\right)-f\left(w y^{\mathrm{t}(w)}\right)\right| e^{-t \phi^{n}\left(w x^{\mathrm{t}(w)}\right)} .
\end{aligned}
$$

Let $\left\{w_{m}\right\}_{m=1}^{\infty}$ be any enumeration of $W^{*}$. Then

$$
\lim _{m \rightarrow+\infty} d\left(\left(w_{m} x^{\mathrm{t}(w)}, w_{m} y^{\mathrm{t}(w)}\right)=0,\right.
$$

so that, since $f$ is continuous,

$$
\lim _{m \rightarrow+\infty} f\left(w_{m} x^{\mathrm{t}(w)}\right)-f\left(w_{m} y^{\mathrm{t}(w)}\right)=0 .
$$


Thus, since each set $W_{n}$ is finite, given $\epsilon>0$, there exists $N \geq 1$ such that if $w \in W_{n}$ and $n \geq N$ then $\left|f\left(w x^{\mathrm{t}(w)}\right)-f\left(w y^{\mathrm{t}(w)}\right)\right|<\epsilon$. Thus,

$$
\begin{aligned}
\left|\zeta_{f, x}(t)-\zeta_{f, y}(t)\right| & \leq 2 t\|f\|_{\infty}^{t}(N-1)+\epsilon t\|f\|_{\infty}^{t-1}\left(\sum_{n=N}^{\infty} \sum_{w \in W_{n}} e^{-t \phi^{n}\left(w x^{\mathrm{t}(w)}\right)}\right) \\
& \leq 2 t\|f\|_{\infty}^{t}(N-1)+\epsilon t\|f\|_{\infty}^{t-1} \eta_{1}(t) .
\end{aligned}
$$

Hence, $\zeta_{f, y}(t)$ converges provided $\zeta_{f, x}(t)$ converges and we have the estimate

$$
\begin{aligned}
\lim _{t \rightarrow 1+}(t-1)\left(\zeta_{f, x}(t)-\zeta_{f, y}(t)\right) & \leq \epsilon \lim _{t \rightarrow 1+}(t-1) \eta_{1}(t) \\
& =\epsilon\left(\frac{1}{\int \phi d \mu}\right)\left(\sum_{j=1}^{k}\left(L_{-\phi} \chi_{j}\right)\left(x^{j}\right)\right)
\end{aligned}
$$

Since $\epsilon>0$ is arbitrary, this shows that

$$
\lim _{t \rightarrow 1+}(t-1) \zeta_{f, x}(t)=\lim _{t \rightarrow 1+}(t-1) \zeta_{f, y}(t) .
$$

A similar argument for $\zeta_{f, z}(t)$ completes the proof of the claim.

To complete the proof, we notice that, as $t \rightarrow 1+$, we have

$$
\begin{aligned}
f\left(w x^{\mathrm{t}(w)}\right)^{t}-f\left(w x^{\mathrm{t}(w)}\right) & =f\left(w x^{\mathrm{t}(w)}\right)\left(f\left(w x^{\mathrm{t}(w)}\right)^{t-1}-1\right) \\
& =f\left(w x^{\mathrm{t}(w)}\right)\left(t-1+O\left((t-1)^{2}\right)\right) .
\end{aligned}
$$

Thus

$$
\zeta_{f, x}(t)-\eta_{f}(t)=\left(t-1+O\left((t-1)^{2}\right)\right) \eta_{f}(t),
$$

so that $\zeta_{f, x}(t)$ converges for $t>1$ and

$$
\lim _{t \rightarrow 1+}(t-1) \zeta_{f, x}(t)=\lim _{t \rightarrow 1+}(t-1) \eta_{f}(t)
$$

as required.

Proof of Theorem 2.1. We need to show that, whenever $f \in C(\Lambda, \mathbb{R})$ with $f \geq 0$, we have

$$
\lim _{n \rightarrow+\infty} \frac{1}{\log n} \sum_{k=1}^{n} a_{k}(f)=c_{\phi} \int f d \mu,
$$

where $\left\{a_{k}(f)\right\}_{k=1}^{\infty}$ are the eigenvalues of $\pi(f)\left|D_{\phi}\right|^{-1}$, counted with multiplicity and written in decreasing order, and where

$$
c_{\phi}=\frac{2}{\int \phi d \mu} \sum_{j=1}^{k} \sum_{T x=x^{j}} e^{-\phi(x)} \chi_{j}(x)=\frac{2}{\int \phi d \mu} \sum_{j=1}^{k}\left(L_{-\phi} \chi_{j}\right)\left(x^{j}\right) .
$$


First, suppose that $f \in C^{\alpha}(\Lambda, \mathbb{R})$ and that $f \geq 0$. Lemma 6.2 and Lemma 6.4 show that $\zeta_{f}(t)$ converges for $t>1$ and diverges for $t=1$. Thus $\pi(f)|D|^{-1} \in$ $\mathscr{L}^{1, \infty}(H)$. It follows immediately from Lemma 6.1 and Lemma 6.4 that

$$
\lim _{n \rightarrow+\infty} \frac{1}{\log n} \sum_{k=1}^{n} a_{k}(f)=\lim _{t \rightarrow 1+}(t-1) \zeta_{f}(t)=c_{\phi} \int f d \mu .
$$

Now suppose $f \in C(\Lambda, \mathbb{R})$ and $f \geq 0$. Given $\epsilon>0$, we may choose $g_{1}, g_{2} \in$ $C^{\alpha}(\Lambda, \mathbb{C})$ such that $0 \leq g_{1} \leq f \leq g_{2}$ and

$$
\int f d \mu-\epsilon \leq \int g_{1} d \mu \leq \int g_{2} d \mu \leq \int f d \mu+\epsilon .
$$

Then we have

$$
\begin{aligned}
c_{\phi}\left(\int f d \mu-\epsilon\right) & \leq c_{\phi} \int g_{1} d \mu=\lim _{t \rightarrow 1+}(t-1) \zeta_{g_{1}}(t) \\
& \leq \liminf _{t \rightarrow 1+}(t-1) \zeta_{f}(t) \leq \limsup _{t \rightarrow 1+}(t-1) \zeta_{f}(t) \\
& \leq \lim _{t \rightarrow 1+}(t-1) \zeta_{g_{2}}(t)=c_{\phi} \int g_{2} d \mu \\
& \leq c_{\phi}\left(\int f d \mu-\epsilon\right) .
\end{aligned}
$$

Since $\epsilon>0$ is arbitrary, the required convergence result holds for $f$.

\section{References}

[1] S. Albeverio, D. Guido, A. Ponosov, and S. Scarlatti, Singular traces and compact operators. J. Funct. Anal. 137 (1996), 281-302. Zbl 0864.47008 MR 1387512

[2] V. Baladi, Positive transfer operators and decay of correlations. Adv. Ser. Nonlinear Dynam. 16, World Scientific, Singapore 2000. Zbl 1012.37015 MR 1793194

[3] R. Bhatia and K. R. Parthasarathy, Lectures on functional analysis. Part I: Perturbation by bounded operators. ISI Lecture Notes 3, Macmillan Co. of India Ltd., New Delhi 1978. Zbl 0451.47020 MR 506158

[4] R. Bowen, Equilibrium states and the ergodic theory of Anosov diffeomorphisms. 2nd revised ed., Lecture Notes in Math. 470, Springer-Verlag, Berlin 2008. Zbl 1172.37001 MR 2423393

[5] E. Christensen and C. Ivan, Spectral triples for AF $C^{*}$-algebras and metrics on the Cantor set. J. Operator Theory 56 (2006), 17-46. Zbl 1111.46052 MR 2261610

[6] F. Cipriani and J.-L. Sauvageot, Fredholm modules on P.C.F. self-similar fractals and their conformal geometry. Comm. Math. Phys. 286 (2009), 541-558. Zbl 1190.28003 MR 2472035 
[7] A. Connes, Noncommutative geometry. Academic Press, San Diego, CA, 1994. Zbl 0818.46076 MR 1303779

[8] A. Connes, Geometry from the spectral point of view. Lett. Math. Phys. 34 (1995), 203-238. Zbl 1042.46515 MR 1345552

[9] A. Connes, Noncommutative geometry and reality. J. Math. Phys. 36 (1995), 6194-6231. Zbl 0871.58008 MR 1355905

[10] J. Dixmier, Existence de traces non normales. C. R. Acad. Sci. Paris Sér. A 262 (1966), 1107-1108. Zbl 0141.12902 MR 0196508

[11] K. Falconer and T. Samuel, Dixmier traces and coarse multifractal analysis. Ergodic Theory Dynam. Systems 31 (2011), 369-381. Zbl 1236.28006 MR 2776380

[12] D. Guido and T. Isola, Fractals in noncommutative geometry. In Mathematical physics in mathematics and physics (Siena, 2000), Fields Inst. Commun. 30, Amer. Math. Soc., Providence, RI, 2001, 171-186. Zbl 1140.46330 MR 1867554

[13] D. Guido and T. Isola, Dimensions and singular traces for spectral triples, with applications to fractals. J. Funct. Anal. 203 (2003), 362-400. Zbl 1031.46081 MR 2003353

[14] D. Guido and T. Isola, Dimensions and spectral triples for fractals in $\mathbb{R}^{N}$. In Advances in operator algebras and mathematical physics, Theta Ser. Adv. Math. 5, Theta, Bucharest 2005, 89-108. Zbl 1199.46160 MR 2238285

[15] T. Kato, Perturbation theory for linear operators. Classics Math., Springer-Verlag, Berlin 1995. Zbl 0836.47009 MR 1335452

[16] M. L. Lapidus and C. Pomerance, The Riemann zeta-function and the one-dimensional Weyl-Berry conjecture for fractal drums. Proc. London Math. Soc. (3) 66 (1993), 41-69. Zbl 0739.34065 MR 1189091

[17] S. Lord, A. Sedaev, and F. Sukochev, Dixmier traces as singular symmetric functionals and applications to measurable operators. J. Funct. Anal. 224 (2005), 72-106. Zbl 1081.46042 MR 2139105

[18] I. C. Palmer, Riemannian geometry of compact metric spaces. PhD Thesis, Georgia Institute of Technology, Atlanta 2010.

[19] W. Parry and M. Pollicott, Zeta functions and the periodic orbit structure of hyperbolic dynamics. Astérisque 187-188 (1990). Zbl 0726.58003 MR 1085356

[20] J. Pearson and J. Bellissard, Noncommutative Riemannian geometry and diffusion on ultrametric Cantor sets. J. Noncommut. Geom. 3 (2009), 447-480. Zbl 1181.46056 MR 2511637

[21] D. Ruelle, Thermodynamic formalism. 2nd ed., Cambridge Math. Lib., Cambridge University Press, Cambridge 2004. Zbl 1062.82001 MR 2129258

[22] T. Samuel, A Commutative noncommutative fractal geometry. PhD Thesis, St Andrews University, St Andrews 2010.

http://research-repository.st-andrews.ac.uk/handle/10023/1710

[23] R. Sharp, Periodic orbits of hyperbolic flows. In G. A. Margulis, On some aspects of the theory of Anosov systems, Springer Monogr. Math., Springer-Verlag, Berlin 2004. Zbl 1140.37010 MR 2035655 
[24] J. C. Várilly, An introduction to noncommutative geometry. EMS Ser. Lect. Math., European Mathematical Society (EMS), Zürich 2006. Zbl 1097.58004 MR 2239597

[25] P. Walters, An introduction to ergodic theory. Grad. Texts in Math. 79, Springer-Verlag, New York 1982. Zbl 0475.28009 MR 648108

Received September 29, 2010; revised November 15, 2010

R. Sharp, School of Mathematics, University of Manchester, Oxford Road, Manchester M13 9PL, UK; current address: Mathematics Institute, University of Warwick, Coventry CV4 7AL, UK

E-mail: R.J.Sharp@warwick.ac.uk 